\title{
Relative Strain in Cu-Nb Composite Wound Wire
}

\author{
R. E. Goddard ${ }^{1}$, K. H. Han ${ }^{1}$, and D. N. Nguyen ${ }^{2}$ \\ 1. National High Magnetic Field Laboratory, Florida State University, Tallahassee, FL USA \\ 2. Pulsed Field Facility (NHMFL), Los Alamos National Laboratory, Los Alamos, NM USA
}

Copper-matrix composite wires with niobium filaments provide high strength and high conductivity [1-2] for winding high-field pulse magnets [3]. As the wire is wound around different diameters, both the transverse cross-section of the wire and the niobium sub-elements are deformed because of differential stress. Each sub-element cross-section area change can be a related to local strain and thus be mapped to give a detailed internal strain distribution [4].

Three coils of $6.4,7.9$, and 12.7 millimeters in diameter were wound with the same rectangular (3.0 by 5.8 millimeters) wires (Fig.1) according to the same procedure (e.g., winding tension and speed). The samples were sectioned to give a transverse axial cross-section on the coil and on the nonwound portion (Fig.2). The samples were encased in conductive mounting medium and polished. Analysis was done in a Zeiss 1540 EsB cross beam Field Emission Gun Scanning Electron Microscope (FEGSEM). Every effort was made to section the coil normal to the transverse crosssection but due to the winding pitch most samples probably have some distortion. All areas of coil cross-sections were thus normalized to the non-wound cross-section area. The transverse rectangular cross-section shape is deformed during winding into a keystone with an expanded inner dimension and a contracted outer dimension (Fig. 2). The smaller the inner coil diameter the greater the distortion between the inside edge and the outside edge as measured by the width edges angular change (Fig. 2). Individual filaments were correlated (Fig. 3) normalized filament area changes were calculated and mapped onto the cross-section surface (Fig. 4).

Because of the homogeneity of the non-wound filament areas (Fig. 4) the slight pitch of the wires during manufacturing caused very little initial strain difference. Large observed area differences are attributed to fabrication of a few half filaments. The two smaller diameter windings show a much greater diversity of filament area size among different filaments. This indicates a larger amount of stress as expected. It is also observed in the area change. However, the $7.9 \mathrm{~mm}$ winding contained the least amount of strain on the inside surface and the no strain line of 0 closest to the inner surface. The smallest diameter winding had the widest variation of strain going from 26 percent in a net tension on the inner surface to -30 percent in net compression on the outer surface. The largest diameter winding of $12.7 \mathrm{~mm}$ had the least amount of strain spread, the least maximum strain, and smoother and more consistent gradation across the width of the wire. When added to the strain induced by energizing the magnet it is possible to calculate the smallest possible diameter that can be used. Of interest is the lack of strain concentration which is an indication of consistent reliable winding technique. These data can be used to better refine modeling by Finite Element Analysis.[5] References:

[1] Deng LP, et al, J. Alloys and Compounds 2014;602:331.

[2] Deng LP, et al, Rare Metal Materials and Engineering 2015;44:1696.

[3] Han K, et al, IEEE Transactions on Applied Superconductivity 2002;12:1176.

[4] Han K, et al, Philosophical magazine 2004;84:2579.

[5] This work has been supported by the National Science Foundation award number DMR-9527035 

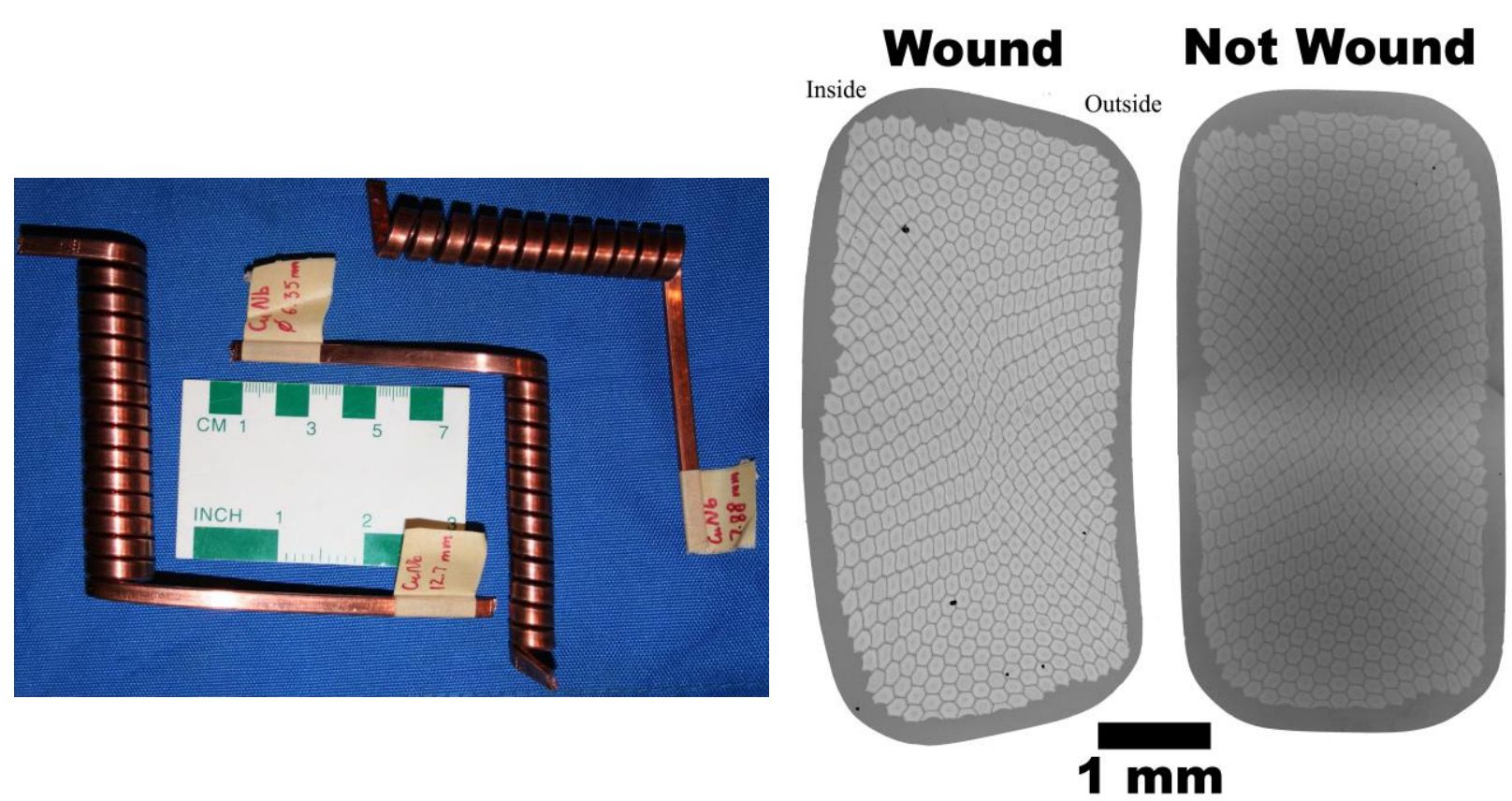

Figure 1. Initial Wire Orientation.

Figure 2. Transverse Wire Cross-Sections.
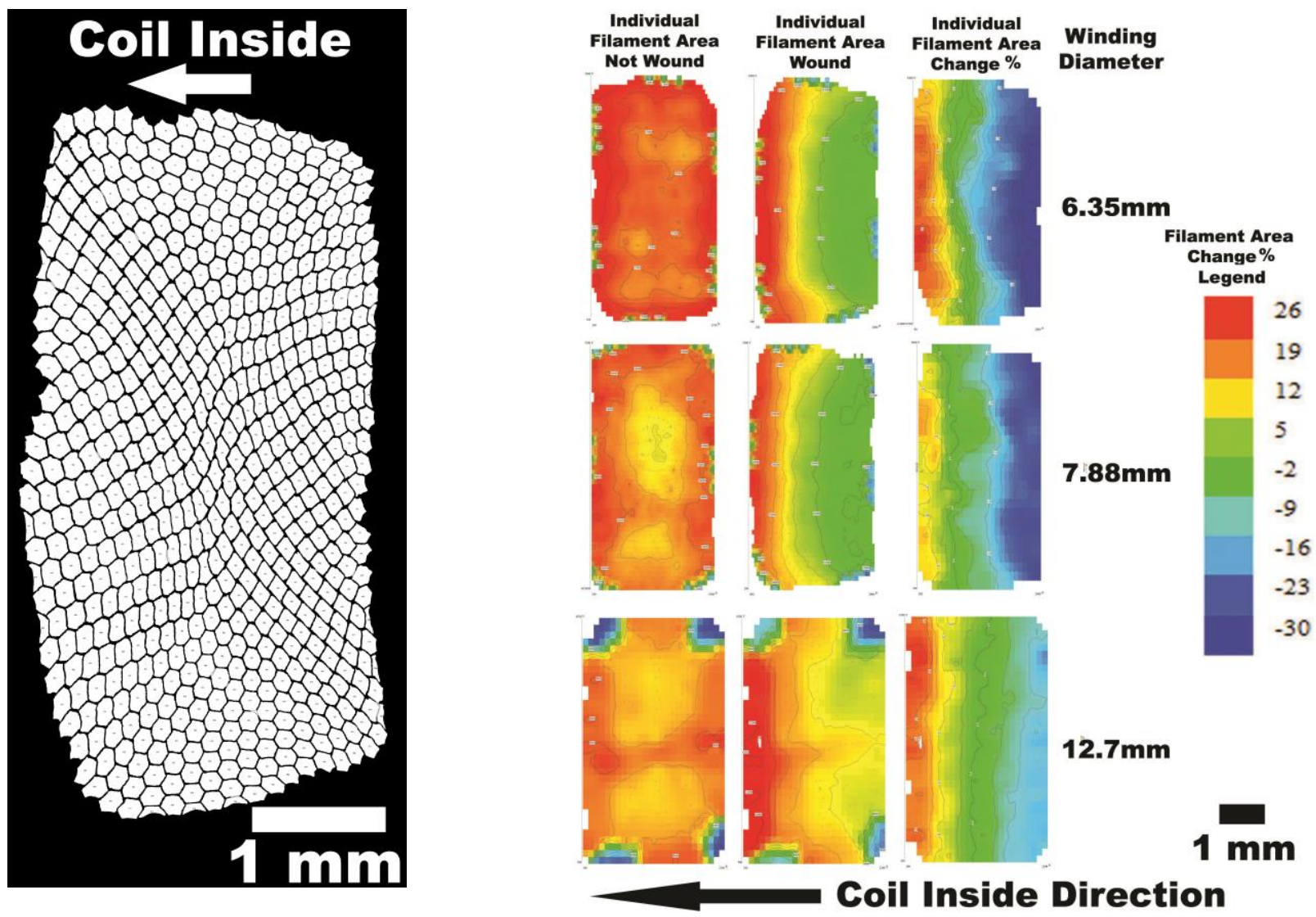

Figure 3. Indentified Filaments.

Figure 4. Contour Maps of Filament Area and Area Change 